\title{
On the Koolen-Park inequality and Terwilliger graphs
}

\author{
Alexander L. Gavrilyuk* \\ Department of Algebra and Topology \\ Institute of Mathematics and Mechanics \\ Ural Division of the Russian Academy of Sciences, Russia \\ alexander.gavriliouk@gmail.com \\ Submitted: Aug 11, 2010; Accepted: Aug 31, 2010; Published: Sep 13, 2010 \\ Mathematics Subject Classifications: 05E30
}

\begin{abstract}
J.H. Koolen and J. Park proved a lower bound for the intersection number $c_{2}$ of a distance-regular graph $\Gamma$. Moreover, they showed that a graph $\Gamma$, for which equality is attained in this bound, is a Terwilliger graph. We prove that $\Gamma$ is the icosahedron, the Doro graph or the Conway-Smith graph if equality is attained and $c_{2} \geqslant 2$.
\end{abstract}

\section{Introduction}

Let $\Gamma$ be a distance-regular graph with degree $k$ and diameter at least 2. Let $c$ be maximal such that, for each vertex $x \in \Gamma$ and every pair of nonadjacent vertices $y, z$ of $\Gamma_{1}(x)$, there exists a $c$-coclique in $\Gamma_{1}(x)$ containing $y, z$. In [1], J.H. Koolen and J. Park showed that the following bound holds:

$$
c_{2}-1 \geqslant \max \left\{\frac{c^{\prime}\left(a_{1}+1\right)-k}{\left(\begin{array}{c}
c^{\prime} \\
2
\end{array}\right)} \mid 2 \leqslant c^{\prime} \leqslant c\right\},
$$

and equality implies that $\Gamma$ is a Terwilliger graph. (For definitions see Sections 2 and 3.)

A similar inequality for a distance-regular graph with a $c$-claw was proved by C.D. Godsil, see [2]. J.H. Koolen and J. Park [1] noted that the bound (1) is met for the three known examples of Terwilliger graphs with $c_{2} \geqslant 2$. We recall that only three examples of distance-regular Terwilliger graphs with $c_{2} \geqslant 2$ are known: the icosahedron, the Doro graph and the Conway-Smith graph.

In this paper, we will show that a distance-regular graph $\Gamma$ with $c_{2} \geqslant 2$, for which equality is attained in (1), is a known Terwilliger graph.

${ }^{*}$ Partially supported by the Russian Foundation for Basic Research (project no. 08-01-00009).

THE ELECTRONIC JOURNAL OF COMBINATORICS 17 (2010), \#R125 


\section{Definitions and preliminaries}

We consider only finite undirected graphs without loops or multiple edges. Let $\Gamma$ be a connected graph. The distance $\mathrm{d}(u, w)$ between any two vertices $u$ and $w$ of $\Gamma$ is the length of a shortest path from $u$ to $w$ in $\Gamma$. The diameter diam $(\Gamma)$ of $\Gamma$ is the maximal distance occurring in $\Gamma$.

For a subset $A$ of the vertex set of $\Gamma$, we will also write $A$ for the subgraph of $\Gamma$ induced by $A$. For a vertex $u$ of $\Gamma$, define $\Gamma_{i}(u)$ to be the set of vertices that are at distance $i$ from $u(0 \leqslant i \leqslant \operatorname{diam}(\Gamma))$. The subgraph $\Gamma_{1}(u)$ is called the local graph of a vertex $u$ and the degree of $u$ is the number of neighbors of $u$, i.e., $\left|\Gamma_{1}(u)\right|$.

For two vertices $u, w \in \Gamma$ with $\mathrm{d}(u, w)=2$, the subgraph $\Gamma_{1}(u) \cap \Gamma_{1}(w)$ is called the $\mu$ subgraph of vertices $u, w$. We say that the number $\mu(\Gamma)$ is well-defined if each $\mu$-subgraph occurring in $\Gamma$ contains the same number of vertices and this number is equal to $\mu(\Gamma)$.

Let $\Delta$ be a graph. A graph $\Gamma$ is locally $\Delta$ if, for all $u \in \Gamma$, the subgraph $\Gamma_{1}(u)$ is isomorphic to $\Delta$. A graph is regular with degree $k$ if the degree of each of its vertices is $k$.

A connected graph $\Gamma$ with diameter $d=\operatorname{diam}(\Gamma)$ is distance-regular if there are integers $b_{i}, c_{i}(0 \leqslant i \leqslant d)$ such that, for any two vertices $u, w \in \Gamma$ with $\mathrm{d}(u, w)=i$, there are exactly $c_{i}$ neighbors of $w$ in $\Gamma_{i-1}(u)$ and $b_{i}$ neighbors of $w$ in $\Gamma_{i+1}(u)$ (we assume that $\Gamma_{-1}(u)$ and $\Gamma_{d+1}(u)$ are empty sets). In particular, a distance-regular graph $\Gamma$ is regular with degree $b_{0}, c_{1}=1$ and $c_{2}=\mu(\Gamma)$. For each vertex $u \in \Gamma$ and $0 \leqslant i \leqslant d$, the subgraph $\Gamma_{i}(u)$ is regular with degree $a_{i}=b_{0}-b_{i}-c_{i}$. The numbers $a_{i}, b_{i}, c_{i}(0 \leqslant i \leqslant d)$ are called the intersection numbers and the array $\left\{b_{0}, b_{1}, \ldots, b_{d-1} ; c_{1}, c_{2}, \ldots, c_{d}\right\}$, is called the intersection array of the distance-regular graph $\Gamma$.

A graph $\Gamma$ is amply regular with parameters $(v, k, \lambda, \mu)$ if $\Gamma$ has $v$ vertices, is regular with degree $k$ and satisfies the following two conditions:

$i)$ for each pair of adjacent vertices $u, w \in \Gamma$, the subgraph $\Gamma_{1}(u) \cap \Gamma_{1}(w)$ contains exactly $\lambda$ vertices;

ii) $\mu=\mu(\Gamma)$ is well-defined.

An amply regular graph with diameter 2 is called a strongly regular graph and is a distance-regular graph. A distance-regular graph is an amply regular graph with parameters $k=b_{0}, \lambda=b_{0}-b_{1}-1$ and $\mu=c_{2}$.

A c-clique $C$ of $\Gamma$ is a complete subgraph (i.e., every two vertices of $C$ are adjacent) of $\Gamma$ with exactly $c$ vertices. We say that $C$ is a clique if it is a $c$-clique for certain $c$. A coclique $C$ of $\Gamma$ is an induced subgraph of $\Gamma$ with empty edge set. We say a coclique is a c-coclique if it has exactly $c$ vertices.

Let $\Gamma$ be a strongly regular graph with parameters $(v, k, \lambda, 1)$. There are integers $r$ and $s$ such that the local graph of each vertex of $\Gamma$ is the disjoint union of $r$ copies of the $s$-clique. Furthermore, $v=1+r s+s^{2} r(r-1), k=r s$ and $\lambda=s-1$. The set of strongly regular graph with parameters $\left(1+r s+s^{2} r(r-1), r s, s-1,1\right)$ is denoted by $\mathcal{F}(s, r)$. 
Any graph of $\mathcal{F}(1, r)$, i.e., a strongly regular graph with $\lambda=0$ and $\mu=1$, is called a Moore strongly regular graph. It is well known (see Ch. 1 [3]) that any Moore strongly regular graph has degree $2,3,7$ or possibly 57 . The graphs with degree 2,3 and 7 are the pentagon, the Petersen graph and the Hoffman-Singleton graph, respectively. It is still unknown whether there exists a Moore graph with degree 57.

Lemma 2.1 If $\mathcal{F}(s, r)$ is a nonempty set of graphs, then $s+1 \leqslant r$.

Proof. Let $\Gamma$ be a graph of $\mathcal{F}(s, r)$. We can choose vertices $u$ and $w$ from $\Gamma$ with $\mathrm{d}(u, w)=2$. Let $x$ be a vertex of $\Gamma_{1}(u) \cap \Gamma_{1}(w)$. Then the subgraph $\Gamma_{1}(w)-\left(\Gamma_{1}(x) \cup\{x\}\right)$ contains a coclique of size at most $r-1$. Let us consider an $s$-clique of $\Gamma_{1}(u)-\Gamma_{1}(w)$ on vertices $y_{1}, y_{2}, . ., y_{s}$. The subgraph $\Gamma_{1}(w) \cap \Gamma_{1}\left(y_{i}\right)(1 \leqslant i \leqslant s)$ contains a single vertex $z_{i}$. The vertices $z_{1}, z_{2}, . ., z_{s}$ are mutually nonadjacent and distinct. Hence, $s \leqslant r-1$. The lemma is proved.

\section{Terwilliger graphs}

In this section we give a definition of Terwilliger graphs and some useful facts concerning them.

A Terwilliger graph is a connected non-complete graph $\Gamma$ such that $\mu(\Gamma)$ is well-defined and each $\mu$-subgraph occurring in $\Gamma$ is a complete graph (hence, there are no induced quadrangles in $\Gamma$ ). If $\mu(\Gamma)>1$, then, for each vertex $u \in \Gamma$, the local graph of $u$ is also a Terwilliger graph with diameter 2 and $\mu\left(\Gamma_{1}(u)\right)=\mu(\Gamma)-1$.

For an integer $\alpha \geqslant 1$, the $\alpha$-clique extension of a graph $\bar{\Gamma}$ is the graph $\Gamma$ obtained from $\bar{\Gamma}$ by replacing each vertex $\bar{u} \in \bar{\Gamma}$ by a clique $U$ with $\alpha$ vertices, where, for any $\bar{u}, \bar{w} \in \bar{\Gamma}$, $u \in U$ and $w \in W, \bar{u}$ and $\bar{w}$ are adjacent if and only if $u$ and $w$ are adjacent.

Lemma 3.1 Let $\Gamma$ be an amply regular Terwilliger graph with parameters $(v, k, \lambda, \mu)$, where $\mu>1$. Then there is a number $\alpha$ such that the local graph of each vertex of $\Gamma$ is the $\alpha$-clique extension of a strongly regular Terwilliger graph with parameters $(\bar{v}, \bar{k}, \bar{\lambda}, \bar{\mu})$, where

$$
\bar{v}=k / \alpha, \bar{k}=(\lambda-\alpha+1) / \alpha, \bar{\mu}=(\mu-1) / \alpha,
$$

and $\alpha \leqslant \bar{\lambda}+1$. In particular, if $\bar{\lambda}=0$, then $\alpha=1$.

Proof. The result follows from [3, Theorem 1.16.3].

There are only three amply regular Terwilliger graphs known with $\mu \geqslant 2$. All of them are distance-regular and are characterized by theirs intersection arrays. The three examples are:

(1) the icosahedron with intersection array $\{5,2,1 ; 1,2,5\}$ is locally pentagon graph;

(2) the Doro graph with intersection array $\{10,6,4 ; 1,2,5\}$ is locally Petersen graph;

(3) the Conway-Smith graph with intersection array $\{10,6,4,1 ; 1,2,6,10\}$ is locally Petersen graph. 
In [4], A. Gavrilyuk and A. Makhnev showed that a distance-regular locally HoffmanSingleton graph has intersection array $\{50,42,9 ; 1,2,42\}$ or $\{50,42,1 ; 1,2,50\}$ and hence it is a Terwilliger graph. Whether there exist graphs with these intersection arrays is an open question.

Lemma 3.2 Let $\Gamma$ be a Terwilliger graph. Suppose that, for an integer $\alpha \geqslant 1$, the local graph of each vertex of $\Gamma$ is the $\alpha$-clique extension of a Moore strongly regular graph $\Delta$. Then $\alpha=1$ and one of the following holds:

(1) $\Delta$ is the pentagon and $\Gamma$ is the icosahedron;

(2) $\Delta$ is the Petersen graph and $\Gamma$ is the Doro graph or the Conway-Smith graph;

(3) $\Delta$ is the Hoffman-Singleton graph or a Moore graph with degree 57; in both cases, the diameter of $\Gamma$ is at least 3.

Proof. It is easy to see that the graph $\Gamma$ is amply regular. By Lemma 3.1, we have $\alpha=1$. Statements (1) and (2) follow from [3, Proposition 1.1.4] and [3, Theorem 1.16.5], respectively.

If the graph $\Delta$ is the Hoffman-Singleton graph and the diameter of $\Gamma$ is 2 , then $\Gamma$ is strongly regular with parameters $(v, k, \lambda, \mu)$, where $k=50, \lambda=7$ and $\mu=2$. By [3, Theorem 1.3.1], the eigenvalues of $\Gamma$ are $k$ and the roots of the quadratic equation $x^{2}+(\mu-\lambda) x+(\mu-k)=0$. The roots of the equation $x^{2}-5 x-48=0$ are not integers, a contradiction. In the remaining case, when $\Delta$ is regular with degree 57 , we get the same contradiction. The lemma is proved.

The next lemma will be used in the proof of Theorem 4.2 (see Section 4).

Lemma 3.3 Let $\Gamma$ be a strongly regular Terwilliger graph with parameters $(v, k, \lambda, \mu)$. Suppose that, for an integer $\alpha \geqslant 1$, the local graph of each vertex of $\Gamma$ is the $\alpha$-clique extension of a strongly regular graph with parameters $(\bar{v}, \bar{k}, \bar{\lambda}, \bar{\mu})$. Then the inequality $\bar{k}-\bar{\lambda}-\bar{\mu}>1$ implies that $k-\lambda-\mu>1$.

Proof. We have $k=\alpha(1+\bar{k}+\bar{k}(\bar{k}-\bar{\lambda}-1) / \bar{\mu}), \lambda=\alpha \bar{k}+\alpha-1$ and $\mu=\alpha \bar{\mu}+1$. If $\bar{k}-\bar{\lambda}-\bar{\mu}>1$, then $\bar{k}(\bar{k}-\bar{\lambda}-1) / \bar{\mu}>\bar{k}$ and this implies that $k-\lambda-\mu=\alpha(\bar{k}(\bar{k}-\bar{\lambda}-1) / \bar{\mu}-\bar{\mu})>$ $\alpha(\bar{k}-\bar{\mu})>\alpha(\bar{\lambda}+1) \geqslant 1$.

\section{The Koolen-Park inequality}

In this section, we consider bound (1) and classify distance-regular graphs with $c_{2} \geqslant 2$, for which this bound is attained.

The next statement is a slight generalization of Proposition 3 from [1], which was formulated by J.H. Koolen and J. Park for distance-regular graphs. We generalize it to amply regular graphs. (Our proof is similar to the proof in [1], but we give it for the convenience of the reader.) 
Proposition 4.1 Let $\Gamma$ be an amply regular graph with parameters $(v, k, \lambda, \mu)$, and let $c \geqslant 2$ be maximal such that, for each vertex $x \in \Gamma$ and every pair of nonadjacent vertices $y, z$ of $\Gamma_{1}(x)$, there exists a c-coclique in $\Gamma_{1}(x)$ containing $y, z$. Then

$$
\mu-1 \geqslant \max \left\{\frac{c^{\prime}(\lambda+1)-k}{\left(\begin{array}{c}
c^{\prime} \\
2
\end{array}\right)} \mid 2 \leqslant c^{\prime} \leqslant c\right\},
$$

and, if equality is attained, then $\Gamma$ is a Terwilliger graph.

Proof. Let $\Gamma_{1}(x)$ contain a coclique $C^{\prime}$ on vertices $y_{1}, y_{2}, \ldots, y_{c^{\prime}}, c^{\prime} \geqslant 2$. Since d $\left(y_{i}, y_{j}\right)=2$, it follows that $\left|\Gamma_{1}(x) \cap \Gamma_{1}\left(y_{i}\right) \cap \Gamma_{1}\left(y_{j}\right)\right| \leqslant \mu-1$ holds for all $i \neq j$. Then, by the inclusionexclusion principle,

$$
\begin{gathered}
k=\left|\Gamma_{1}(x)\right| \geqslant\left|\cup_{i=1}^{c^{\prime}}\left(\Gamma_{1}(x) \cap\left(\Gamma_{1}\left(y_{i}\right) \cup\left\{y_{i}\right\}\right)\right)\right| \\
\geqslant \sum_{i=1}^{c^{\prime}}\left|\Gamma_{1}(x) \cap\left(\Gamma_{1}\left(y_{i}\right) \cup\left\{y_{i}\right\}\right)\right|-\sum_{1 \leqslant i<j \leqslant c^{\prime}}\left|\Gamma_{1}(x) \cap \Gamma_{1}\left(y_{i}\right) \cap \Gamma_{1}\left(y_{j}\right)\right| \\
\geqslant c^{\prime}(\lambda+1)-\left(\begin{array}{c}
c^{\prime} \\
2
\end{array}\right)(\mu-1) .
\end{gathered}
$$

So,

$$
\mu-1 \geqslant \frac{c^{\prime}(\lambda+1)-k}{\left(\begin{array}{c}
c^{\prime} \\
2
\end{array}\right)} .
$$

Note that equality in (2) implies that the inclusion $\Gamma_{1}(x) \subseteq \cup_{i=1}^{c^{\prime}}\left(\Gamma_{1}\left(y_{i}\right) \cup\left\{y_{i}\right\}\right)$ holds and we have $\left|\Gamma_{1}(x) \cap \Gamma_{1}\left(y_{i}\right) \cap \Gamma_{1}\left(y_{j}\right)\right|=\mu-1$ for all $i \neq j$.

Let $c$ be the maximal number satisfying the condition of Proposition 4.1. Then

$$
\mu-1 \geqslant \max \left\{\frac{c^{\prime}(\lambda+1)-k}{\left(\begin{array}{c}
c^{\prime} \\
2
\end{array}\right)} \mid 2 \leqslant c^{\prime} \leqslant c\right\} .
$$

We may assume that for an integer $c^{\prime \prime}$, where $2 \leqslant c^{\prime \prime} \leqslant c$, (3) turns into equality, i.e.,

$$
\mu-1=\frac{c^{\prime \prime}(\lambda+1)-k}{\left(\begin{array}{c}
c^{\prime \prime} \\
2
\end{array}\right)}=\max \left\{\frac{c^{\prime}(\lambda+1)-k}{\left(\begin{array}{c}
c^{\prime} \\
2
\end{array}\right)} \mid 2 \leqslant c^{\prime} \leqslant c\right\} .
$$

We will show that $c=c^{\prime \prime}$. For a vertex $x \in \Gamma$ and nonadjacent vertices $y, z \in \Gamma_{1}(x)$, there exists a $c$-coclique $C$ in $\Gamma_{1}(x)$ containing $y, z$. Equality (4) implies that, for any subset of vertices $\left\{y_{1}, y_{2}, \ldots, y_{c^{\prime \prime}}\right\} \subseteq C$, we have $\Gamma_{1}(x) \subseteq \cup_{i=1}^{c^{\prime \prime}}\left(\Gamma_{1}\left(y_{i}\right) \cup\left\{y_{i}\right\}\right)$. However, if $c^{\prime \prime}<c$, then $C \not \subset \cup_{i=1}^{c^{\prime \prime}}\left(\Gamma_{1}\left(y_{i}\right) \cup\left\{y_{i}\right\}\right)$, a contradiction.

Hence, $c=c^{\prime \prime}$ and we have $\left|\Gamma_{1}(x) \cap \Gamma_{1}(y) \cap \Gamma_{1}(z)\right|=\mu-1$ for every pair of nonadjacent vertices $y, z \in \Gamma_{1}(x)$ and for all $x \in \Gamma$. This implies that each $\mu$-subgraph in $\Gamma$ is a clique of size $\mu$ and $\Gamma$ is a Terwilliger graph.

We call inequality (3) the $\mu$-bound.

It is easy to check that the three known Terwillger graphs with $\mu \geqslant 2$ (see Section 3 ) have equality in the $\mu$-bound.

Our main theorem is to show that the only Terwilliger graphs with $\mu \geqslant 2$ and equality in the $\mu$-bound are the three known examples (of Section 3). 
Theorem 4.2 Let $\Gamma$ be an amply regular graph with parameters $(v, k, \lambda, \mu)$, and let $\mu>1$. If the $\mu$-bound is attained, then $\mu=2$ and $\Gamma$ is the icosahedron, the Doro graph or the Conway-Smith graph.

Proof. By Proposition 4.1, the graph $\Gamma$ is a Terwilliger graph and, by Lemma 3.1, there is an integer $\alpha \geqslant 1$ such that the local graph of each vertex of $\Gamma$ is the $\alpha$-clique extension of a strongly regular Terwilliger graph with parameters $(\bar{v}, \bar{k}, \bar{\lambda}, \bar{\mu})$. By Lemma 3.1, we have $k=\alpha \bar{v}, \lambda=\alpha \bar{k}+(\alpha-1)$ and $\mu=\alpha \bar{\mu}+1$.

By the assumption on $\Gamma$, for a vertex $u \in \Gamma$, the local graph of $u$ contains a $c$-coclique, for which equality is attained in the $\mu$-bound, i.e.,

$$
\mu-1=\alpha \bar{\mu}=\frac{c(\lambda+1)-k}{\left(\begin{array}{l}
c \\
2
\end{array}\right)}=\frac{c(\alpha \bar{k}+(\alpha-1)+1)-\alpha \bar{v}}{\left(\begin{array}{l}
c \\
2
\end{array}\right)}=\alpha \frac{c(\bar{k}+1)-\bar{v}}{\left(\begin{array}{l}
c \\
2
\end{array}\right)}
$$

and

$$
\bar{\mu}=\frac{c(\bar{k}+1)-\bar{v}}{\left(\begin{array}{l}
c \\
2
\end{array}\right)} .
$$

Hence, $c$ satisfies the following quadratic equation:

$$
c^{2} \bar{\mu}-c(\bar{\mu}+2(\bar{k}+1))+2 \bar{v}=0,
$$

in other words,

$$
c=\frac{(\bar{\mu}+2(\bar{k}+1)) \pm \sqrt{(\bar{\mu}+2(\bar{k}+1))^{2}-8 \bar{v} \bar{\mu}}}{2 \bar{\mu}} .
$$

This implies that

$$
(\bar{\mu}+2(\bar{k}+1))^{2} \geqslant 8 \bar{v} \bar{\mu} .
$$

Let the subgraph $\Gamma_{1}(u)$ be isomorphic to the $\alpha$-clique extension of a strongly regular Terwilliger graph with parameters $(\bar{v}, \bar{k}, \bar{\lambda}, \bar{\mu})$, say $\Delta$. The cardinality of the vertex set of $\Delta$ is $\bar{v}=1+\bar{k}+\bar{k}(\bar{k}-\bar{\lambda}-1) / \bar{\mu}$, hence

$$
\begin{gathered}
(\bar{\mu}+2(\bar{k}+1))^{2} \geqslant 8(\bar{\mu}+\bar{k} \bar{\mu}+\bar{k}(\bar{k}-\bar{\lambda}-1)), \\
\bar{\mu}^{2}+4 \geqslant 4 \bar{\mu}+4 \bar{k} \bar{\mu}+4 \bar{k}^{2}-8 \bar{k} \bar{\lambda}-16 \bar{k} .
\end{gathered}
$$

Further,

$$
\begin{gathered}
(\bar{\mu} / 2)^{2}+1 \geqslant \bar{\mu}+\bar{k} \bar{\mu}+\bar{k}^{2}-2 \bar{k} \bar{\lambda}-4 \bar{k}, \\
((\bar{\mu} / 2)-(\bar{k}+1))^{2} \geqslant 2 \bar{k}(\bar{k}-\bar{\lambda}-1) .
\end{gathered}
$$

Let us first consider the case $\bar{\mu}=1$. There are integers $s, r$ such that $\Delta \in \mathcal{F}(s, r)$ and $\bar{k}=r s, \bar{\lambda}=s-1$. If $\bar{k}-\bar{\lambda}-1 \geqslant \bar{k} / 2+1$, then $2 \bar{k}(\bar{k}-\bar{\lambda}-1) \geqslant 2 \bar{k}(\bar{k} / 2+1)=\bar{k}^{2}+2 \bar{k}$. It follows from (5) that $(\bar{k}+1 / 2)^{2} \geqslant \bar{k}^{2}+2 \bar{k}$ and hence $1 / 4 \geqslant \bar{k}$, which is impossible. Therefore, $\bar{k}-\bar{\lambda}-1<\bar{k} / 2+1$, i.e., $\bar{k}<2(\bar{\lambda}+2)$. Substituting the expressions for $\bar{k}$ and $\bar{\lambda}$ into the previous inequality, we get $r s<2(s+1)$. By Lemma 2.1, we have $s+1 \leqslant r$. Hence, $s+1 \leqslant r<2(s+1) / s$ and it follows that $s=1, r \in\{2,3\}$ and $\Delta$ is the pentagon 
or the Petersen graph. As we already checked that the three examples in Lemma $3.2(i)$ and (ii) satisfy equality in the $\mu$-bound, Theorem 4.2 follows in this case from Lemma 3.2 .

Now we may assume $\bar{\mu}>1$. Since $\bar{\mu}<\bar{k}$, the left-hand side of (5) is at most $\bar{k}^{2}$. On the other hand, if $\bar{k}-\bar{\lambda}-1>\bar{k} / 2$, then the right-hand side of (5) is greater than $2 \bar{k} \bar{k} / 2=\bar{k}^{2}$, which is impossible. Hence, we have $\bar{k}-\bar{\lambda}-1 \leqslant \bar{k} / 2$, i.e., $\bar{k} \leqslant 2(\bar{\lambda}+1)$.

Since $\bar{\mu}>1$, there is an integer $\alpha_{1} \geqslant 1$ such that, for a vertex $w \in \Delta$, the subgraph $\Delta_{1}(w)$ is the $\alpha_{1}$-clique extension of a strongly regular Terwilliger graph, say $\Sigma$, with parameters $\left(v_{1}, k_{1}, \lambda_{1}, \mu_{1}\right)$, where $v_{1}=\frac{\bar{k}}{\alpha_{1}}, k_{1}=\frac{\bar{\lambda}-\left(\alpha_{1}-1\right)}{\alpha_{1}}, \mu_{1}=\frac{\bar{\mu}-1}{\alpha_{1}}$. Then the inequality $\bar{k} \leqslant 2(\bar{\lambda}+1)$ is equivalent to the inequality $v_{1} \leqslant 2\left(k_{1}+1\right)$ and the cardinality of the vertex set of $\Sigma$ is

$$
v_{1}=1+k_{1}+k_{1} \frac{\left(k_{1}-\lambda_{1}-1\right)}{\mu_{1}} .
$$

Further, $v_{1} \leqslant 2\left(k_{1}+1\right)$ implies that

$$
\frac{k_{1}\left(k_{1}-\lambda_{1}-1\right)}{\mu_{1}} \leqslant k_{1}+1,
$$

So

$$
k_{1}-\lambda_{1}-1 \leqslant \mu_{1}\left(1+1 / k_{1}\right)<\mu_{1}+1
$$

and

$$
k_{1}<\lambda_{1}+\mu_{1}+2 .
$$

If $\mu_{1}=1$, then, for certain $s_{1}, r_{1}$, we have $k_{1}=r_{1} s_{1}$ and $\lambda_{1}=s_{1}-1$. It follows from (6) that $r_{1} s_{1}<s_{1}-1+1+2=s_{1}+2, r_{1}<1+2 / s_{1}$ and $s_{1}=1, r_{1}=2$. Hence, the graph $\Delta_{1}(w)$ is the $\alpha_{1}$-clique extension of the pentagon. By Lemma 3.2, the graph $\Delta$ is the icosahedron and the diameter of $\Gamma_{1}(u)$ is 3 , which is impossible because $\Gamma$ is a Terwilliger graph.

Hence, $\mu_{1}>1$. Let us consider a sequence of strongly regular graphs $\Sigma_{1}=\Sigma$, $\Sigma_{2}, \ldots, \Sigma_{h}, h \geqslant 2$, such that, for an integer $\alpha_{i+1} \geqslant 1$, the local graph of a vertex in $\Sigma_{i}$ is the $\alpha_{i+1}$-clique extension of a strongly regular Terwilliger graph $\Sigma_{i+1}$ with parameters $\left(v_{i+1}, k_{i+1}, \lambda_{i+1}, \mu_{i+1}\right), 1 \leqslant i<h$ and $\mu\left(\Sigma_{h}\right)=1$, i.e., $\Sigma_{h} \in \mathcal{F}\left(s_{h}, r_{h}\right)$ for certain $s_{h}, r_{h}$. Such a sequence exists by Lemma 3.1.

Assuming $s_{h}>1$, we get $k_{h}-\lambda_{h}-\mu_{h}=r_{h} s_{h}-\left(s_{h}-1\right)-1=s_{h}\left(r_{h}-1\right)>1$. According to Lemma 3.3, we have $k_{i}-\lambda_{i}-\mu_{i}>1$ for all $1 \leqslant i \leqslant h-1$, which contradicts (6). Hence, $s_{h}=1$ and $\Sigma_{h}$ is a Moore strongly regular graph. By Lemma 3.2, the diameter of $\Sigma_{h-1}$ is at least 3 , and this contradiction completes the proof.

\section{Acknowledgements}

I would like to thank Ekaterina Vasilyeva and Maxim Ananyev (for the help in translation of this paper to English) and Prof. Jack Koolen for his comments, which greatly improved the paper. I would also like to thank J. Park for his careful reading of the paper.

This work was partially supported by the Russian Foundation for Basic Research (project no. 08-01-00009). 


\section{References}

[1] J.H. Koolen, J. Park: Shilla distance-regular graphs. // arXiv:0902.3860 [math.CO]

[2] C.D. Godsil: Geometric distance-regular covers // New Zealand J. Math. 22 (1993), 3138.

[3] A.E. Brouwer, A.M. Cohen, A. Neumaier: Distance-Regular Graphs. SpringerVerlag, Berlin Heidelberg New York, 1989.

[4] A.L. Gavrilyuk, A.A. Makhnev: Locally Hoffman-Singleton Distance-Regular Graphs // to appear. 